\title{
ACTUALITÉS AUC
}

\section{Andrew MacNeily, M. D., FRCSC}

\section{Président de l'AUC}

Citer comme suit : MacNeily AE. Avant de tirer ma révérence. Can Urol Assoc J 2021;15(6):E305-6. http://dx.doi.org/10.5489/cuaj.7415

L'AUC a pour mandat de promouvoir les plus hautes normes dans les soins urologiques pour les Canadiens et de faire avancer l'art et la science de l'urologie.

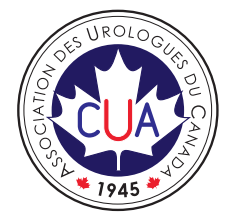

a dernière fois qu'un président de l'AUC est resté en poste pendant deux ans remonte au mandat du $\mathrm{D}^{r}$ Charles Robson, qui avait été prolongé par l'annulation du congrès de 1968 aux Bermudes, en raison d'une crise financière. Mon propre mandat a été prolongé à cause d'une crise d'un autre genre. Néanmoins, contre toute attente, les deux dernières années ont vu de nombreux développements positifs au sein de l'AUC grâce au travail acharné du Bureau corporatif et de nombreux urologues bénévoles. Voici quelques exemples :

- Nous avons maintenant un Comité d'urologie en milieu non académique (CUMNA) très solide qui a une voix au sein de la direction de l'AUC et du Comité des finances. S'appuyant sur les bases jetées par le Dr Frank Papanikolaou, le $\mathrm{D}^{\mathrm{r}}$ Lorne Aaron, président du CUMNA, demeure un porte-parole sage et puissant pour les $70 \%$ des membres de I'AUC qui s'identifient comme étant en milieu non universitaire. Un nouveau programme de mentorat CUMNA-AUC a été lancé afin de fournir un soutien à l'acquisition de nouvelles compétences, tout en minimisant les obstacles financiers pour les mentorés et les mentors.

- Après quatre années de productivité phénoménale en tant que président du Comité des politiques de santé, le $\mathrm{D}^{\mathrm{r}}$ Omar Nazif a passé le flambeau au $\mathrm{D}^{\mathrm{r}}$ Troy Sitland, président par intérim. Troy est parfaitement capable de composer avec les complexités créées par les disparités interprovinciales quant au remboursement, aux ressources hospitalières et au devenir des stagiaires après leurs études, complexités auxquelles son comité s'attaque régulièrement. Merci, Troy, d'avoir accepté cette grande responsabilité.

- La nouvelle politique d'équité, de diversité et d'inclusion a donné naissance à un comité pleinement fonctionnel prenant des mesures concrètes pour régler cette question qui aurait dû être abordée il y a longtemps. La D ${ }^{\text {re }}$ Ashley Cox, nouvelle vice-présidente des communications, a accepté de bonne grâce le poste supplémentaire de responsable de l'EDI. Les membres peuvent s'attendre à une activité importante de la part du comité d'EDI. Suivez-nous de près.

- Notre association a la chance d'avoir une nouvelle secrétaire, la De Marie-Paule Jammal. Elle a été l'assistante du Dr Hassan Razvi au cours de la dernière année et apporte une nouvelle perspective et un regain d'énergie à I'un des postes les plus exigeants du Conseil d'administration de I'AUC. Merci, Marie-Paule!

- Notre situation financière est excellente, grâce aux innovations cumulées de notre Bureau corporatif, du vice-président à l'éducation, le Dr Ricardo Rendon, du trésorier, le Dr Wassim Kassouf, et des présidents des conseils de la FBAUC, les $\mathrm{D}^{\text {rs }}$ Chris French et Anil Kapoor. L'ensemble de leurs connaissances, leur intégrité et leur ténacité ont permis de maintenir l'AUC en bonne santé financière malgré l'incertitude créée par la pandémie.

- Le Bureau corporatif et le Bureau de l'éducation sont passés sans heurt à des plateformes virtuelles pour toutes les formes d'activités de FMC, ce qui a énormément amélioré la tenue de nos activités éducatives. Les cours du soir de 2020 ont connu beaucoup de succès, et le programme pour 2021 semble encore meilleur. Les membres remarqueront également plusieurs changements positifs sur le nouveau site Web, y compris UROpédia, la source de toutes les activités éducatives de l'AUC. Jetez-y un coup d'œil! 
- Au cours des deux dernières années, le président du Comité des guides de pratique, le Dr Bobby Shayegan, a fait un boulot remarquable quand il s'agit de motiver les membres du comité à produire plusieurs guides de pratique et rapports sur les meilleures pratiques; plusieurs autres sont en préparation. Quiconque a déjà fait partie d'un comité de guides de pratique sait à quel point l'élaboration de ces documents peut être une tâche laborieuse. Si ce n'est pas votre cas, mais que vous appréciez les fruits du travail de ce comité, veuillez vous porter volontaire pour y participer.

- $\quad$ Le D Mike Leveridge a succédé au Dr Rob Siemens au poste de rédacteur en chef du JAUC, un changement de leadership des plus réussis. En conséquence, le JAUC continue de prospérer. Le journal a incorporé des crédits de DPC de section 3 pour certains articles, et la conversion à un modèle de publication électronique avant l'impression a considérablement réduit le temps entre l'acceptation et la publication des articles. Le look du JAUC et l'interface du site Web ont été mis à jour, pour une expérience plus conviviale. D'autres changements sont à venir.

- Lorsque le Dr Jerzy Gajewski a pris sa retraite en tant qu'historien, le Dr Michael Leonard a pris la relève sans perdre le rythme. Le grand dévouement de Jerzy à la couverture de notre histoire pendant de nombreuses années avait placé la barre très haute, mais Mike a pris le relais avec la publication régulière de ses "Moments marquants de l'histoire de l'urologie ", qui sont divertissants, informatifs et essentiels pour garder l'histoire de l'AUC vivante.

- Le soutien de I'AUC aux étudiants en urologie n'a jamais été aussi élevé. Des programmes tels que QUEST, la foire de I'urologie, T-Res pour la déclaration des cas et le camp de formation en sciences fondamentales nouvellement revitalisé sont sous la direction du président du Comité des affaires des résidents, le Dr Keith Rourke. De plus, le Comité des résidents et des boursiers offre désormais un apport direct au Conseil d'administration. Et au niveau du premier cycle, le Dr Trustin Domes, président du Comité du cursus de premier cycle en urologie au Canada, a fait un travail fantastique en rassemblant des capsules vidéo sur le thème "Qu'est-ce que I'urologie? " pour aider les futurs urologues à en apprendre davantage sur notre grande spécialité.

Et ça ne s'arrête pas là. Ce n'est qu'un échantillon des nombreux développements positifs survenus pendant mon mandat de président, malgré un environnement de travail des plus difficiles créé par la pandémie. J'aimerais avoir plus d'espace pour parler de tous les succès récents de I'AUC. Je tiens à m'excuser auprès de tous les intervenants que j'ai pu omettre.

Alors que j'entre dans ma septième décennie, il est clair que j'en ai plus de fait qu'il ne m'en reste à faire. Apparemment, je suis maintenant un " ancien » (qui se demande encore au fond ce qu'il va faire quand il sera grand). Pour moi, ces deux années à la présidence de l'AUC ont été à la fois agréables et remplies d'incertitudes et de rebondissements. J'ai été impressionné par l'énergie et la conviction que toutes les personnes impliquées ont consacrées à l'AUC. Je vous remercie tous pour votre soutien, et pour votre opposition réfléchie, à l'occasion. Nous sommes tous très chanceux d'appartenir à l'AUC. S'il vous plaît, offrez de votre temps. Vous ne le regretterez pas.

Cela dit, tout a une fin. Le 30 juin 2021 marque l'inauguration de votre nouveau président, le $\mathrm{D}^{\mathrm{r}}$ Hassan Razvi. Il apporte une main ferme à la barre, avec des années d'expérience dans de multiples fonctions au sein de la direction de l'AUC. Je suis persuadé que vous serez satisfaits de son leadership.

À vous, Hassan. MacNeily, terminé! 